\title{
DOPPLER TOMOGRAPHY OF CHROMOSPHERES AND ACCRETION REGIONS IN ALGOL BINARIES
}

\author{
MERCEDES T. RICHARDS \& GEARY E. ALBRIGHT \\ Department of Astronomy, University of Virginia \\ P.O. Box 3818, Charlottesville, VA 22903-0818, USA
}

\begin{abstract}
The technique of back-projection Doppler tomography has been used to produce the first set of reconstructed images of chromospheric emission sources in the class of Algol-type binaries. The $\mathrm{H} \alpha$ emission sources in the Doppler tomograms include the gas stream, a sub-Keplerian accretion annulus, a quasi-stable accretion disk, and the chromosphere of the cool secondary star. The chromospheric images of several Algol secondaries (e.g., RS Vul, $\beta$ Per, TX UMa, RW Tau) are consistent with that of the RS CVn binary V711 Tau.
\end{abstract}

\section{Introduction}

The class of Algol-type binaries has been studied actively for at least three centuries since the discovery of photometric variability in Algol ( $\beta$ Persei). Since then, there have been extensive photometric and spectroscopic observations of these systems. Optical and ultraviolet spectra, as well as UBV photometry, of these systems suggest the presence of gas flows near the primary star which result from Roche lobe overflow (Olson 1980, 1989; Peters 1984; Etzel, Olson \& Senay 1995; Richards 1992, 1993; Albright \& Richards 1993, 1995). However, the X-ray and radio emission detected from these binaries arise exclusively from magnetic activity associated with the secondary star (Richards \& Albright 1993 and references therein).

The Algol-type binaries contain a hot B-A V primary star and a cooler magnetically active F-K III-IV secondary star. These are semi-detached binary systems in which the less massive secondary has filled its Roche lobe and is transferring gas through the $L_{1}$ point onto the more massive primary star at typically slow mass transfer rates of $\dot{M} \sim 10^{-11}-10^{-7}$ $M_{\odot} \mathrm{yr}^{-1}$. Our general picture of the Algols has been influenced mostly by 
the $\mathrm{H} \alpha$ studies of Peters $(1980,1989)$ and Kaitchuck, Honeycutt \& Schlegel (1985). In the short-period systems $\left(P_{\text {orb }}<6\right.$ days $)$, the gas stream from the secondary does not have enough room to form a stable accretion disk; instead it makes a direct impact with the photosphere of the primary star and forms a time-dependent asymmetric distribution of gas (the accretion annulus) around that star. In the wider long-period Algols $\left(P_{o r b}>6\right.$ days), the stream forms a classical accretion disk around the primary star. However, a combination of the two previous cases is apparent in the intermediate systems with $P_{\text {orb }} \sim 4-6$ days. Moreover, tidal interactions in the short-period binaries result in an increase in the rotational velocity of the secondary which generates enhanced magnetic activity on this star. There is a subsequent decrease in the primary's rotation.

The nature of the accretion structures of these systems is now unfolding with the aid of Doppler tomography. This technique requires complete orbital coverage of each binary to provide fairly accurate results, and the quality of the image improves with the orbital resolution. The more generalized technique of computerized tomography was formulated by the Austrian mathematician Johann Radon (Radon 1917; Herman 1980) and has been used in the field of medicine to reconstruct 3D images of parts of the human body from 2D pictures or "slices" (e.g., CAT scans). However, in astronomy this same technique can be used only to generate $2 \mathrm{D}$ images of the accretion regions in the orbital plane of an eclipsing binary from the 1D information provided by the Doppler radial velocities of the emission lines. In this case, the "slices" are azimuthal views of the binary as seen from different positions in the orbit (orbital phase), and the reconstructed image is displayed in dimensions of velocity and not in the usual Cartesian dimensions. This image can be converted to spatial dimensions only if the velocity field is known.

Doppler tomography was first applied by Marsh et al. (1990) to the $\mathrm{H} \beta$, He I ( $\lambda 4471)$ and He II ( $\lambda 4686)$ spectral lines of the cataclysmic variable U Gem. An atlas of Doppler tomograms of 18 cataclysmic variables was also produced by Kaitchuck et al. (1994). Some of these images display a toroid of emission (an accretion disk) centered on the white dwarf mass gainer. The technique has also been applied to $\mathrm{H} \alpha$ spectra of the Algols by Richards, Albright \& Bowles (1995), Richards, Jones \& Swain (1996), and Albright \& Richards (1995). These studies have shown the presence of gas streams and accretion annuli in the class of Algols as well as some limited evidence of chromospheric emission at $\mathrm{H} \alpha$.

In this work we describe the complex tomographic images of Algol binaries and make comparisions with the image of the magnetically active RS CVn binary V711 Tau to illustrate the evidence for chromospheric emission in the Algols. 


\section{Observations}

The full-orbit $\mathrm{H} \alpha$ CCD spectra of Algol binaries used in this work were obtained with the $0.9 \mathrm{~m}$ coudé feed telescope at Kitt Peak National Observatory (KPNO) and the $1.5 \mathrm{~m}$ telescope at McMath-Pierce Solar Observatory (NSO) from 1990 October to 1994 December. The 1990 and 1991 NSO data were $32 \AA$ wide with a reciprocal dispersion of $2.7 \AA \mathrm{mm}^{-1}$, while the 1992 observations have a spectral field of $73 \AA$ and a reciprocal dispersion of 6.09 $\AA \mathrm{mm}^{-1}$. The KPNO data have a spectral field of $177 \AA$ and a dispersion of $14.8 \AA \mathrm{mm}^{-1}$ (1992 spectra), or a spectral field of $345 \AA$ and a dispersion of $7.0 \AA \mathrm{mm}^{-1}$ (1993 spectra). The coudé feed was used very effectively to cover the orbits of up to 9 binaries during a single 7-night observing run and reduce any long-term variability in the data. As a result, we now have a unique data set which contains nearly complete orbital coverage of several binaries within 3 orbital cycles. Some systems were observed in subsequent years to provide a secular perspective (see Table 1 ). The data obtained

TABLE 1. Properties of Observed Systems

\begin{tabular}{lclllr}
\hline Binary & $P_{\text {orb }}$ (days) & Spectral type & Year & Telescope & Data \\
\hline Algols: & & & & & \\
RW Tau & 2.7688 & B8V + K0IV & 1994 & coudé feed & 30 \\
$\beta$ Per & 2.8673 & B8V + K2IV & 1992 & McMath-Pierce & 135 \\
& & & 1994 & coudé feed & 36 \\
TX UMa & 3.0632 & B8V + G0III-IV & 1992 & McMath-Pierce & 81 \\
& & & 1993 & McMath-Pierce & 114 \\
& & & 1994 & coudé feed & 27 \\
U Sge & \multirow{2.3806}{*}{ B8V + G4III-IV } & 1993 & coudé feed & 106 \\
& & & 1994 & coudé feed & 48 \\
U CrB & 3.4522 & B6V + G0III-IV & 1993 & McMath-Pierce & 161 \\
& & & 1994 & coudé feed & 47 \\
RS Vul & 4.4777 & B5V + G1III & 1993 & coudé feed & 81 \\
SW Cyg & 4.5731 & A2V + K0IV & 1994 & coudé feed & 36 \\
TT Hya & 6.9534 & B9V + G9III & 1994 & coudé feed & 21 \\
& & & & & \\
RS CVns: & & & & & \\
V711 Tau & 2.8377 & K1IV + G5IV & 1994 & coudé feed & 27 \\
\hline
\end{tabular}

since 1990 October include spectra of over 14 Algols, with limited coverage of 4 others: V505 Sgr, RZ Cas, TW Cas, TV Cas, $\delta$ Lib, RW Tau, TW Dra, $\beta$ Per, TX UMa, U Sge, S Equ, U CrB, RS Vul, SW Cyg, TT Hya, AU Mon, and RY Gem. Table 1 shows a list of the observed systems for which images have been reconstructed. 
In the short-period Algols, the emission from the circumstellar gas is weak relative to the luminosity of the stars, so it is difficult to study the gas flows unless the photospheric component is removed. To achieve this end, the combined theoretical (Kurucz ATLAS9) LTE stellar photospheric $\mathrm{H} \alpha$ line profile was subtracted from the observed line profile to produce "difference profiles" at each orbital phase, with line strengths scaled to the total continuum flux of each system (cf. Richards 1993). These difference profiles represent the contributions of all non-photospheric gas flows in the binary, namely those produced by Roche lobe overflow and the chromosphere of the magnetically active cool secondary star. The difference profiles typically show blends of emission and absorption, with either single or double-peaked emission at out-of-eclipse phases. Broad absorption profiles are seen during primary eclipse, and often a narrow emission feature is superposed on the absorption near the middle of this eclipse.



Figure 1. Appearance of observed $\mathrm{H} \alpha$ line profiles with orbital phase (left panel): $\mathrm{RZ}$ Oph $\left(P=262^{d}\right)$, AU Mon $\left(P=11.1^{d}\right)$, TT Hya $\left(P=6.9^{d}\right)$, SW Cyg $\left(P=4.5^{d}\right)$, and U Sge $\left(P=3.4^{d}\right)$. Difference profiles for TT Hya, SW Cyg, and U Sge are also shown (right panel).

In the case of V711 Tau, no difference profiles were calculated because strong emission (up to 1.3 times the continuum flux) was detected in the observed $\mathrm{H} \alpha$ spectra. 


\section{Doppler Tomography}

The $\mathrm{H} \alpha$ emission sources were found with a back-projection Doppler tomography code (Kaitchuck et al. 1994; Robinson, Marsh \& Smak 1993: eqns. [9]-[15]). The basic procedure is to convert the wavelength scale of each spectrum to a velocity scale relative to the rest wavelength of the $\mathrm{H} \alpha$ line in the rest frame of the binary. Then, within the tomography code, the profiles are passed through a fast-Fourier filter with a Gaussian of FWHM $=3 \sigma$, where $\sigma$ is the velocity spacing of the pixels in the spectra. The intensity at each point in velocity space $\left(V_{x}, V_{y}\right)$ is then calculated from the entire data set with equal weights applied to each orbital phase. The result is a tomogram of the emission sources in the orbital plane, but displayed in velocity coordinates.

The Doppler maps of several Algol binaries were generated from their $\mathrm{H} \alpha$ difference profiles (see Fig. 2). These maps were made from the out-ofeclipse data listed in Table 1. In addition, the influence of chromospheric emission on the Doppler map was illustrated with the aid of the observed $\mathrm{H} \alpha$ emission profiles (not difference profiles) of V711 Tau. Since we do not fully understand the sources of the emission and are unable to separate these sources, the Doppler velocities $\left(V_{x}, V_{y}\right)$ cannot be immediately converted to the corresponding spatial or Cartesian $(x, y)$ coordinates. So we use several labels on the maps to assist us (cf. Richards et al. 1996). The emission regions arising from an accretion disk or gas stream in the binary have expected shapes in a Doppler tomogram. Double-peaked line profiles would map into a disk-like structure centered on the mass gainer (outermost solid and dashed circles). Moreover, emission from the gas stream would be readily identified because its free-fall path in the rotating frame of the binary is plotted in the tomogram (see Fig. 2). The location where the gas stream comes in contact with the stellar photosphere is also shown (asterisk). This also shows the chain of points in velocity space representing the Keplerian velocity field at each point along the path of the gas stream (dot-dashed trajectory). If stream material is being deflected into the main Keplerian flow in a shocked region at some point along the gas stream, then emission should be seen in the region in velocity space between the free-fall and Keplerian velocities at that geometrical point.

\section{Results and Discussion}

To date, the technique of Doppler Tomography has been used to analyze the $\mathrm{H} \alpha$ difference profiles of RW Tau, $\beta$ Per, TX UMa, U Sge, U CrB, RS Vul, SW Cyg, and TT Hya (Richards et al. 1995, 1996; Albright \& Richards 1995). A preliminary study of the Doppler tomograms suggests that there are as many as 7 sources of emission, the strongest of which arise 

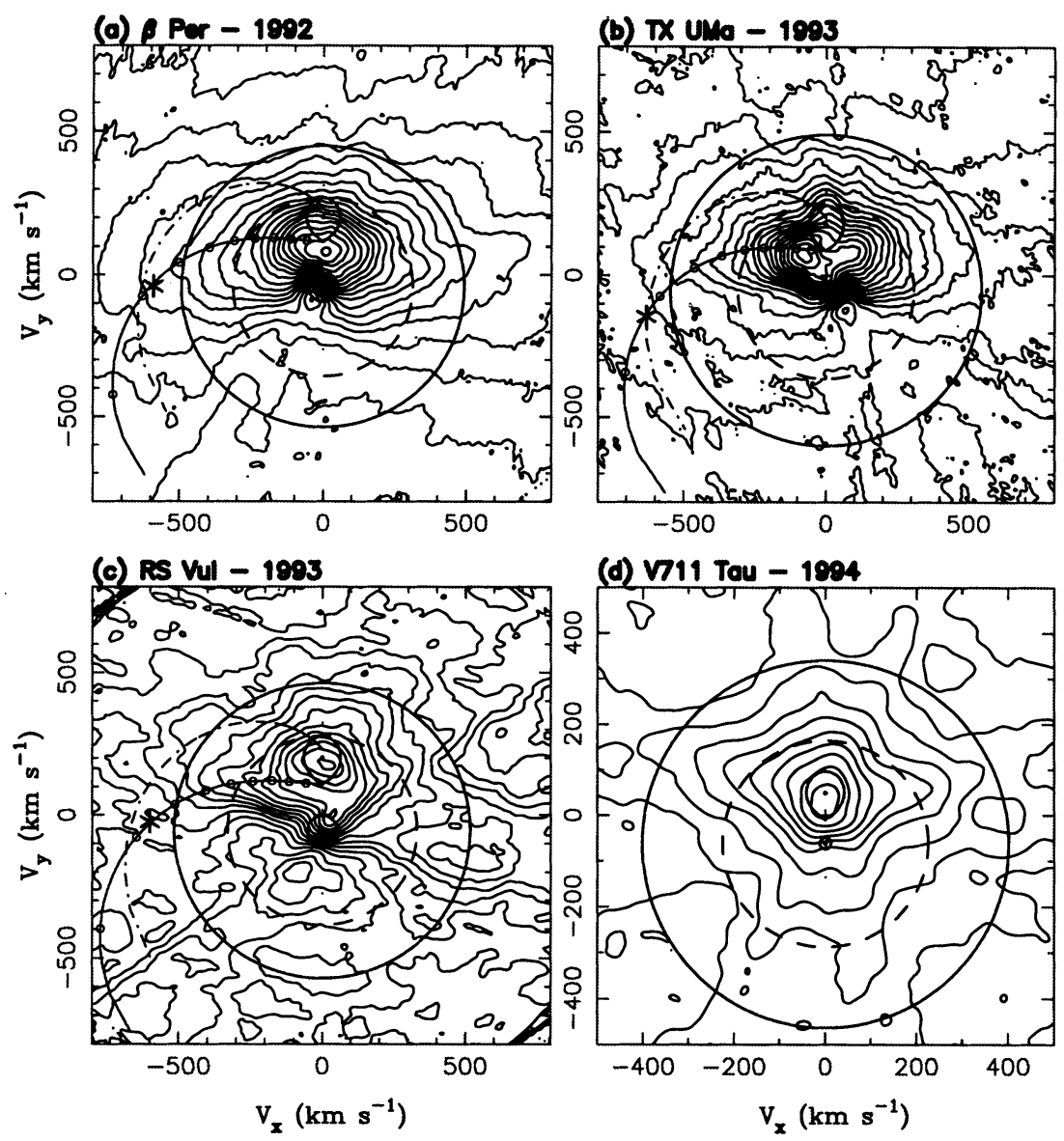

Figure 2. Contour Doppler maps of magnetically active systems: $\beta$ Per (1992), TX UMa (1993), RS Vul (1993), and V711 Tau (1994).

in a gas stream and an accretion annulus or quasi-stable disk surrounding the primary star. In particular, the tomograms of RS Vul (1993), U Sge (1994), U CrB (1994) and $\beta$ Per (1992) showed distinct elongated emission along the gravitational free fall path of the gas stream trajectory from the $L_{1}$ point towards the primary star. The collimation of the gas stream was more pronounced in U CrB (1994) than in the other systems (Richards et al. 1995). Doppler maps of some systems, e.g., U CrB (1993) and U Sge (1993), showed quasi-stable accretion structures around the mass gaining star in addition to the gas stream. So it is apparent that the Algols with $P_{\text {orb }}<4.6$ days periodically change from a state where the gas stream is strong to one in which the accretion disk is strong, but both components are always present. For the binaries with $P_{\text {orb }}>4.6$ days, e.g., SW Cyg 
(1994) and TT Hya (1994), the tomogram displayed a prominent, almost Keplerian disk which is similar to those found in the cataclysmic variables.

In this work, we describe the evidence for another yet another emission source. In the Algols, this source has almost the same velocity as the magnetically active star, hence may arise from the chromosphere of that star. This source was prominent in RS Vul (1993), TX UMa $(1992,1993,1994)$, $\beta$ Per $(1992,1994)$, and RW Tau (1994). Figure 2 shows that the strongest emission source in RS Vul is centered on the velocity of the cool G1 III star, while in the remaining Algol systems the chromospheric source is weaker relative to other emission sources. The tomogram of RW Tau (1994) is similar to those of $\beta$ Per. Moreover, the tomogram of TX UMa (1992) suggests that the gas stream does not follow the gravitational trajectory. One explanation is that the magnetic field associated with the secondary in this system is strong enough to dominate the gravitational process. A Doppler tomogram of the $\mathrm{H} \alpha$ emission in the RS CVn binary V711 Tau was also made. The orbital coverage of this binary was not as good as we would have liked, but this system provided a useful check on the back-projection procedure. As expected, the tomogram of V711 Tau displays no evidence of mass transfer via Roche lobe overflow, but contains a strong source centered on the more active $\mathrm{K} 1 \mathrm{IV}$ component, with a less intense source extending to the less active, fainter G5 IV companion. There is no obvious double chromospheric source in this case because of the relative strengths of the $\mathrm{K}$ to G chromospheres. While RS Vul contains a strong gas stream source, the similarities between this Algol binary and V711 Tau are quite interesting.

This work provides the first evidence of chromospheric emission in the class of Algol secondaries. However, it should be noted that this emission source was found by assuming that all the non-photospheric gas was optically thin, and hence that the photospheric and chromospheric contributions were merely additive; an assumption which is not correct in the optically thick chromospheric environment. Nevertheless, since we had no a priori knowledge of possible chromospheric contributions, we could not have modelled this chromospheric contribution as accurately as for RS CVn binaries or magnetically active stars. The mere detection of emission associated with the Algol secondaries is a good preliminary result even though our results cannot be used to get a precise estimate of the relative contribution of the chromosphere to the combined emission sources in each binary. When compared with the $\mathrm{H} \alpha$ emission from V711 Tau, the Algol chromospheres are 0.2 to 6.2 times as powerful since they contribute $\sim 4 \%$ $-30 \%$ of the continuum flux at this wavelength. The chromospheric source in RS Vul is the strongest of all the systems studied, and is 6 times stronger than that in V711 Tau. These results are in agreement with the evidence of magnetic activity from $\mathrm{X}$-ray and radio data. 
A continuous long-term radio survey of $\beta$ Per and V711 Tau has been in effect since 1995 January to study the flaring timescales of these systems at $2 \mathrm{GHz}$ and $8 \mathrm{GHz}$ (Richards, Waltman, Foster \& Ghigo 1995). The observations are being collected with the Naval Research Labs (NRL) interferometer at Green Bank Radio Observatory (USA). These flaring timescales may provide a clue to the processes which result in the extreme variability of the $\mathrm{H} \alpha$ emission in systems like $\mathrm{U} \mathrm{CrB}$ and $\mathrm{U}$ Sge, or those which influence the gas stream trajectory in TX UMa.

Both 2-D and 3-D numerical hydrodynamical simulations of these binaries are now underway to study the structure of the accretion flows in the absence of magnetic fields (e.g., Blondin, Richards \& Malinkowski 1995; Ratliff \& Richards 1995). The simulations are now being illustrated in both Cartesian and velocity coordinates for easy comparison with the Doppler tomograms. It is hoped that these simulations will allow us to understand better how magnetic fields influence the flow of gas in Algol binaries.

\section{Acknowledgements}

We would like to thank K. Horne for introducing us to the technique of back-projection tomography. This research was partially supported by NSF grant AST 93-15108 and AFOSR grant F49620-92-J-0024.

\section{References}

Albright, G. E. and Richards, M. T. (1993) ApJ, 414, 830

Albright, G. E. and Richards, M. T. (1995), submitted to ApJ

Blondin, J. M., Richards, M. T. and Malinkowski, M. (1995) ApJ, 445, 939

Etzel, P. B, Olson, E. C. \& Senay, M. C. (1995), AJ, 109, 1269

Herman, G. T. 1980, Image Reconstruction From Projections: The Fundamentals of Computerized Tomography (New York: Academic)

Kaitchuck, R. H. (1989), Space Sci. Rev., 50, 51

Kaitchuck, R. H., Honeycutt, R. K. \& Schlegel, E. M. (1985), PASP, 97, 1178

Kaitchuck, R. H., Schlegel, E. M., Honeycutt, R. K., et al. (1994), ApJS, 93, 519

Marsh, T. R. \& Horne, K. (1988), MNRAS, 235, 269

Marsh, T. R., Horne, K., Schlegel, E. M., et al. (1990), ApJ, 364, 637

Olson, E. C. (1980), ApJ, 241, 257

Olson, E. C. (1989), Space Sci. Rev., 50, 23

Peters, G. J. (1989), Space Sci. Rev., 50, 9

Peters, G. J. \& Polidan, R. S. (1984), $A p J, \mathbf{2 8 3}, 745$

Radon, J. (1917), reprinted in Proc. Symp. in Appl. Math. (1983), 27, 71

Ratliff, M. A., \& Richards, M. T. (1995), in preparation

Richards, M. T. (1993), ApJ Supp., 86, 255

Richards, M. T. \& Albright, G. E. (1993), ApJ Supp., 88, 199

Richards, M. T., Albright, G. E. \& Bowles, L. M. (1995), ApJ Lett., 438, L103

Richards, M. T., Jones, R. D. \& Swain, M. A. (1996), ApJ, 459 (March 1), in press

Richards, M. T., Waltman, E. B., Foster, R. S., \& Ghigo, F. 1995, in preparation

Robinson, E. L., Marsh, T. R., \& Smak, J. I. (1993), in Accretion Disks in Compact Stellar Systems, ed. J. C. Wheeler (Singapore: World Scientific), p. 75 